\title{
A Prática Clínica Baseada em Evidências. Parte II - Buscando as Evidências em Fontes de Informação(*)
}

\section{Evidence Based Clinical Practice. Part II - Searching Evidence Databases}

\author{
Wanderley Marques Bernardo, Moacyr Roberto Cuce Nobre, Fábio Biscegli Jatene
}

\section{RESUMO}

A busca da resposta a uma questão clínica bem construída pode ser realizada em inúmeras fontes de informação científica. $\mathrm{Na}$ dependência de onde obtemos a informação, a decisão clínica não será sustentada pelas melhores evidências disponíveis na atualidade, expondo o paciente a um risco desnecessário. A busca pode ser realizada em bases primárias, que disponibilizam os trabalhos originais, cabendo ao leitor o ônus de selecionar e analisar criticamente a validade de seus resultados. A busca pode também ser realizada em bases secundárias, que economizam o tempo do leitor na seleção metodológica e avaliação crítica. Entre as bases primárias, recomendamos o Medline e o SciELO, onde a busca pode ter início com a utilização das palavras-chaves, obtidas na construção da pergunta estruturada segundo o acrônimo P.I.C.O., em seguida, organizadas com a adição dos booleanos AND, OR ou NOT. Entre as bases secundárias que acrescem qualidade científica na seleção das evidências apresentadas, algumas fornecem respostas fundamentadas em trabalhos inviduais, como o ACP Journal Club, o Evidence Based Medicine e o InfoPoems, outras abordam questões clínicas organizadas em forma de livro, como o Clinical Evidence e o UpToDate. A Cochrane Review apresenta evidências de boa qualidade a partir de revisões sistemáticas de ensaios clínicos preferencialmente aleatorizados. A obtenção de estudos que respondem a uma questão clínica faz parte do atendimento que procura integrar a melhor evidência científica às necessidades de atendimento do paciente. Para tanto, conta com recursos tecnológicos, como os PDAs, Palmtops e Notebooks, cada vez mais adaptados para trazer a última informação divulgada pela Internet que auxilia na tomada de decisão clínica à beira do leito.

Palavras-chave: Medicina Baseada em Evidência. Ciência da Informação. Sistemas de Suporte para Decisões Clínicas. Epidemiologia Clínica. PubMed. Revisões Sistemáticas.

\begin{abstract}
The inadequacy of most of traditional sources for medical information, like textbook and review article, do not sustained the clinical decision based on the best evidence current available, exposing the patient to a unnecessary risk. Although not integrated around clinical problem areas in the convenient way of textbooks, current best evidence from specific studies of clinical problems can be found in an increasing number of Internet and electronic databases. The sources that have already undergone rigorous critical appraisal are classified as secondary information sources, others that provide access to original article or abstract, as primary information source, where the quality assessment of the article rely on the clinician oneself. The most useful primary information source are SciELO, the online collection of Brazilian scientific journals, and Medline, the most comprehensive database of the USA National Library of Medicine, where the search may start with use of keywords, that were obtained at the structured answer construction (P.I.C.O), with the addition of boolean operators "AND", "OR", "NOT". Between the secondary information sources, some of them provide critically appraised articles, like ACP Journal Club, Evidence Based Medicine and InfoPOEMs, others provide evidences organized as online texts, such as "Clinical Evidence" and "UpToDate», and finally, Cochrane Library are composed by systematic reviews of randomized controlled trials. To get studies that could answer the clinical question is part of a mindful practice, that is, becoming quicker and quicker and dynamic with the use of PDAs, Palmtops and Notebooks. [Rev Assoc Med Bras 2004; 50(1): 104-8].
\end{abstract}

Keywords: Evidence based medicine. Information science. Clinical decision support systems. Clinical epidemiology. PubMed. Systematic reviews.

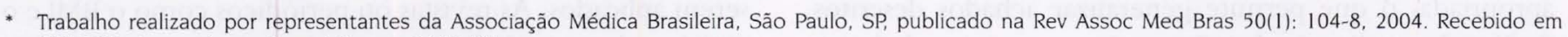
02/12/03. Aprovado, após revisão, em 26/01/04
} 
O processo de encontrar a resposta apropriada à dúvida surgida no atendimento do paciente depende da forma como estruturamos as partes deste processo. Como já descrito na primeira parte ${ }^{(1)}$ desta série de artigos, utilizamos a forma estruturada de formular a pergunta sintetizada pelo acrônimo P.I.C.O., onde o P corresponde ao paciente ou população, I de intervenção ou indicador, $\mathrm{C}$ de comparação ou controle, e O de "outcome" ou desfecho. A partir da pergunta estruturada identificamos as palavraschave ou descritores que irão constituir a base da busca da evidência nas diversas bases de dados disponíveis.

No entanto, antes de descrevermos as principais bases de dados biomédicas, bem como algumas das estratégias de busca nas quais podemos aplicar os diferentes componentes da pergunta estruturada, devemos considerar as diferenças entre uma revisão narrativa e uma revisão sistemática, já que esta última, quando elaborada com rigor metodológico, se constitui na melhor evidência disponível.

As revisões tradicionais incluem artigos de revisão e livros de texto, que geralmente são narrativas de natureza opinativa, considerados com força de evidência científica precária, já que não podem ser reproduzidos por outros autores. Estes textos são gerados segundo a opinião do autor, que decide quais as informações são mais relevantes, sem explicitar a forma como elas são obtidas. $\mathrm{O}$ autor costuma buscar trabalhos que reforcem o seu ponto de vista, não considerando aqueles que divergem ou têm propostas alternativas, mesmo que produzidos com boa metodologia de pesquisa. Como o autor escolhe de forma arbitrária os artigos de onde provem a informação, as orientações que se depreendem do texto estão sujeitas ao viés de seleção, com grande interferência da percepção subjetiva. Quando diversos textos de natureza opinativa são articulados por um conjunto de autores, habitualmente pertencentes a uma mesma especialidade, ou sociedades afins, compõem-se um documento conhecido como consenso, que costuma atender às composições de natureza corporativa.

Sempre que possível devem ser substituídos por revisões conhecidas como sistemáticas, com ou sem meta-análise, que se utilizam de metodologia reprodutível, explícita, critérios de pesquisa e seleção de informação, de tal forma que outros autores que queiram reproduzir a mesma metodologia podem chegar aos mesmos conteúdo e conclusões. São consideradas de grande força de evidência científica. As informações são criticamente avaliadas por metodologia apropriada, o que permite generalizar achados descritos, facilitando a aplicação da informação no contexto do leitor. Ou seja, as informações, geradas no grupo de pacientes estudados, podem ser aplicadas ao paciente que se pretende orientar. A meta-análise ou análise dos dados combinados a partir dos diversos trabalhos selecionados estima mais precisamente os resultados, aumentando ainda mais a força de evidência.

O exemplo classicamente citado nesta comparação entre revisão narrativa e revisão sistemática está relacionado ao uso de trombolíticos no infarto agudo do miocárdio ${ }^{(2)}$. Este é um estudo de meta-análise cumulativa, na qual os dados são submetidos a uma nova meta-análise sempre que um novo trabalho sobre o mesmo assunto é publicado. Combinando os resultados de seis trabalhos publicados entre o início dos anos 60 e o início dos anos 70, a meta-análise comprova o evidente benefício do uso de trombolíticos no infarto do miocárdio, enquanto somente cerca de 15 anos mais tarde os livros de texto escritos por especialistas passam a recomendar o uso rotineiro. Quantas vidas poderiam ter sido salvas nestes 15 anos se a tomada de decisão médica no tratamento do infarto contasse com fontes atualizadas de informação de maior confiabilidade?

As revisões sistemáticas estão preferencialmente disponíveis em bases de dados denominadas secundárias ou préselecionadas ${ }^{(3)}$, como a Cochrane Database of Systematic Reviews, e devem se constituir no início da busca da resposta, sobretudo quando a questão clínica é bem focada ou específica. As fontes de informações são classificadas em primárias se os trabalhos publicados de forma integral ou resumida encontram-se na sua forma original, como no MedLine, Lilacs, a maioria dos periódico médicos, como os brasileiros reunidos no portal SciELO. As fontes são consideradas secundárias quando as informações originais são reproduzidas, comentadas e avaliadas criticamente, de acordo com a metodologia preconizada pela epidemiologia clínica, como no ACP Journal Club, Evidence Based Medicine, InfoPOEMs. Para as situações clínicas que são freqüentes na nossa atividade, como a angina instável ou doenças tromboembólicas, para as quais surgem novas informações todos os dias, é melhor fazer a busca em bases primárias que as divulgam em primeira mão, com a desvantagem que deveremos dedicar mais tempo na avaliação crítica da validade de seus resultados ${ }^{(4)}$. Nas outras situações, que apresentam ritmo de atualização mais lento, como por exemplo a arterite temporal, podemos economizar o nosso tempo fazendo a busca em bases secundárias que já apresentam os resultados avaliados criticamente, prontos para serem aplicados. As revistas ou periódicos como o BMJ e o JAMA embora se dediquem prioritariamente a publicação de trabalhos originais, mantêm sessões dedicadas a comentar 
trabalhos pré-avaliados ou a divulgar a metodologia da avaliação crítica. As revisões sistemáticas, embora se utilizem de dados de trabalhos já publicados, apresentam características metodológicas que resultam em informações originais, e não devem ser confundidas com as fontes secundárias onde os trabalhos originais são avaliados individualmente. Nas Tabelas 1,2 e 3 podem ser encontradas as diferentes fontes de informação.

As fontes primárias podem ser editadas em papel ou em versões informatizadas, a base de dados MedLine é a contrapartida online da Index Medicus, como o EMBASE é da Exerpta Médica. As bases de dados informatizadas e acessadas pela Internet têm se constituído na principal fonte de busca de informação. As fontes primárias são mais úteis para localização de informações no desenvolvimento de trabalhos de pesquisa ou divulgação científica, enquanto as fontes secundárias servem para aplicação prática, economizando o tempo que seria gasto na seleção e avaliação crítica da qualidade das informações. Em outras palavras, as fontes primárias servem primordialmente às atividades de pesquisa, elaboração de diretrizes ou à necessidade de informação mais recente para aplicação clínica, enquanto as fontes secundárias servem primordialmente aos profissionais que exercem atividades clínicas, precisam de informação confiável e usam a maior parte do seu tempo para cuidar da saúde das pessoas.

Existem fontes de informação que antecedem a publicação final de resultados de trabalhos científicos em andamento e poderiam ser classificadas como pré-primárias. O exemplo mais extenso é o "Current Controlled Trials", que tem acesso livre através do endereço http:// www.controlled-trials.com/. É caracterizado por um metabuscador, que recupera ensaios clínicos randomizados em andamento por todo o mundo, e atualizado constantemente, contendo aproximadamente mais de 15 mil registros de ensaios em andamento. Além do próprio registro, oferece mais de 250 conecções, ou links, pela rede da Internet, para outras páginas que registram ensaios em andamento.

TABELA 1

FONTES PRIMÁRIAS DE INFORMAČ̃̃O

\begin{tabular}{|c|c|}
\hline $\begin{array}{l}\text { MEDLINE (as mesmas do Index Medicus, medicina, biologia e saúde, início 1966) } \\
\text { EMBASE (as mesmas do Excerpta Medica, medicina, biologia e saúde em geral) }\end{array}$ & $\begin{array}{l}\text { http://www.ncbi.nlm.nih.gov/pubmed/ } \\
\text { http://www.embase.com/ }\end{array}$ \\
\hline CINAHL (enfermagem e outras áreas da saúde) & http://www.cinahl.com/ \\
\hline PsyciNFo (psiquiatria, enfermagem, sociologia, educação) & http://www.psycinfo.com/ \\
\hline CANCERLIT (câncer) & http://www.cancer.gov/search/cancer_literature/ \\
\hline PDQ (câncer) & http://www.nci.nih.gov/cancer_information/pdq/ \\
\hline HealthStar (serviços, tecnologia, administração, e pesquisa em saúde) & $\begin{array}{l}\text { http://www.nlm.nih.gov/databases/ } \\
\text { interim_healthstar.html }\end{array}$ \\
\hline LIFE (current contents - ciências da vida) & http://www.isinet.com/isi/products/cc/ \\
\hline CLIN (current contents - clínica médica) & http://www.isinet.com/isi/products/cc/ \\
\hline BEHA (current contents - ciências sociais e do comportamento) & http://www.isinet.com/isi/products/cc/ \\
\hline PsycLIT (psicologia, medicina, enfermagem, sociologia, educação e outras) & http://www.apa.org/psycinfo/products/pidirect.html \\
\hline DISS (dissertações e teses americanas, canadenses e européias) & http://gateway.ovid.com/ \\
\hline SILABUS (dissertações e teses da USP) & http://www.usp.br/sibi/ \\
\hline BIOSIS (ciências da vida) & http://www.biosis.org/ \\
\hline ERIC (educação) & http://www.askeric.org/Eric/ \\
\hline ADOLEC (saúde na adolescência) & http://www.bireme.br/bvs/P/pbd.htm \\
\hline BDENF (enfermagem) & http://www.medicina.ufmg.br/biblio/bdenf/ \\
\hline WHOLIS (sistema de informação da biblioteca da OMS) & http://www.who.int/library/database/index.en.shtml \\
\hline HAPI (instrumentos para avaliação de saúde e aspectos psicosociais) & http://www.asu.edu/lib/resources/db/hapi.htm \\
\hline LILACS (literatura latino-americana e do caribe em ciências da saúde) & http://www.bireme.br/bvs/P/pbd.htm \\
\hline PAHO (acervo da biblioteca da organização panamericana da saúde) & http://www.bireme.br/bvs/P/pbd.htm \\
\hline
\end{tabular}




\section{TABELA 2}

FONTES SECUNDÁRIAS dE INFORMAÇÃo - PERIÓdICOS QUE PUBliCAM SINOPSES DE ARTIGOS ORIGINAIS, COMENTADOS E avaliados Criticamente SEgundo a metodologia da Medicina Baseada em Evidência

\begin{tabular}{ll}
\hline ACP Journal Club & http://www.acponline.org/ \\
Evidence Based Medicine & http://ebm.bmjjournals.com/ \\
InfoPOEMs & http://www.infopoems.com/ \\
Evidence Based Mental Health & http://www.bmjpg.com/data/ebmhsub.htm \\
Evidence Based Nursing & http://www.hiru.mcmaster.ca/ebn/ \\
Journal of Evidence-Based Health Care & http://www.ihs.ox.ac.uk/ebhc/ \\
Evidence Based Obstetrics \& Gynecology & http://www.harcourt-international.com/journals/ebog \\
Evidence Based Pediatrics \& Child Health & http://www.evidbasedpediatrics.com/ \\
Biblioteca Cochrane & http://cochrane.bireme.br/
\end{tabular}

TABELA 3

FonTES PRIMÁRIAS E SECUNDÁRIAS DE INFORMAÇÃO - PERIÓDICOS QUE PUBLICAM ARTIGOS ORIGINAIS E matérias SObre Medicina Baseada EM EVIDÊNCIA

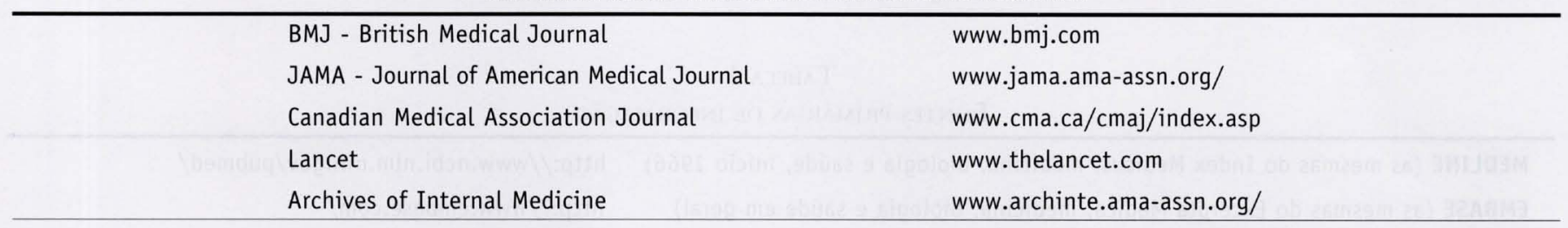

O processo de recuperação da informação em fontes de informação automatizadas, denominadas como base de dados, possibilita a pesquisa de modo interativo, através do computador. A mais utilizada é a base de dados da "National Library of Medicine", responsável pelo Medline, que se encontra em <http:/www.pubmed.gov>. Para tanto, é necessário conhecer a linguagem padronizada pela base de dados, estabelecendo uma associação entre esta linguagem e os componentes da pergunta por nós previamente estruturada.

O Medline é de domínio público e pode ser pesquisado na Internet a partir de portais, páginas de periódicos e de serviços. A busca pode se dar de quatro maneiras principais: a) digitando-se as palavras de interesse diretamente na caixa de diálogo "search Pubmed for"; ou escolhendo-se uma das opções no menu à esquerda no "pubmed services": b) se a opção for "clinical queries", escolha o tipo de estudo e digite as palavras de interesse na caixa de diálogos; c) se a opção for "single citation matcher", escreva dois ou mais dos dados da referência bibliográfica, como sobrenome do autor e nome da revista; d) se a opção for "MeSH Database" escreva a palavra, ou parte da palavra de interesse, que a base oferece inúmeras opções de palavras-chave para sua escolha, incluindo uma árvore classificatória de como as palavras chaves são ordenadas entre si.

$\mathrm{O}$ "MeSH " acrônimo de "Medical Subject Headings" é o vocabulário usado para indexar artigos no MEDLINE e no Index Medicus ${ }^{\circledR}$. Esta última forma é recomendada por tornar a busca mais específica, evitando-se a quantidade excessiva de artigos que não interessam. Mesmo que a preferência de busca recaia sobre as outras três alternativas é recomendável inicialmente selecionar a palavra chave no "MeSH Database".

Ao final podemos utilizar os filtros para tornar ainda mais específica a busca escolhendo opções no "limits". Os mais seletivos no menu "all fields" são: "title" ou "MeSH Major Topic"; "Meta-analysis" ou "Randomized Controlled Trial" no "Publication Types". Pode também ser muito útil limitar a faixa etária no menu "ages", limitar a data da 
publicação no menu "Entrez Date" e os estudos clínicos, no menu "human". Todas as buscas realizadas ficam registradas em "history", através desta página podemos combinar as buscas realizadas anteriormente digitando-se para cada pesquisa o número correspondente, antecedido pelo símbolo do "jogo da velha", unidos pelo operador booleano “AND", por exemplo, \#12 AND \#13. Algumas dicas para busca em bases informatizadas estão na Figura 2 e as principais estratégias de busca na base de dados do PubMed e da Cochrane estão na Tabela 4.

Para exemplificar, retomando o cenário clínico apresentado no primeiro artigo desta série, chegou-se a seguinte questão clínica: Qual a probabilidade de um homem de 48 anos, com exames normais, apresentar recorrência de crise convulsiva após primeiro surto epilético, sem causa aparente? Para encontrarmos a resposta podemos desenvolver a seguinte estratégia de busca, a partir do P.I.C.O. e digitá-la na caixa de diálogo do PubMed: (epilepsy OR seizure OR aura) AND (recurrence OR prognosis) NOT (traumatic OR surg* OR febrile). Ativando os limites: male, middle aged, title, randomized controlled trial foram encontrados 18 artigos submetidos à avaliação crítica da validade interna e quanto à aplicabilidade ao paciente, que resultou na seleção de quatro artigos que respondem à questão do paciente, entre os quais um estudo prognóstico randomizado de excelente qualidade metodológica ${ }^{(5)}$.

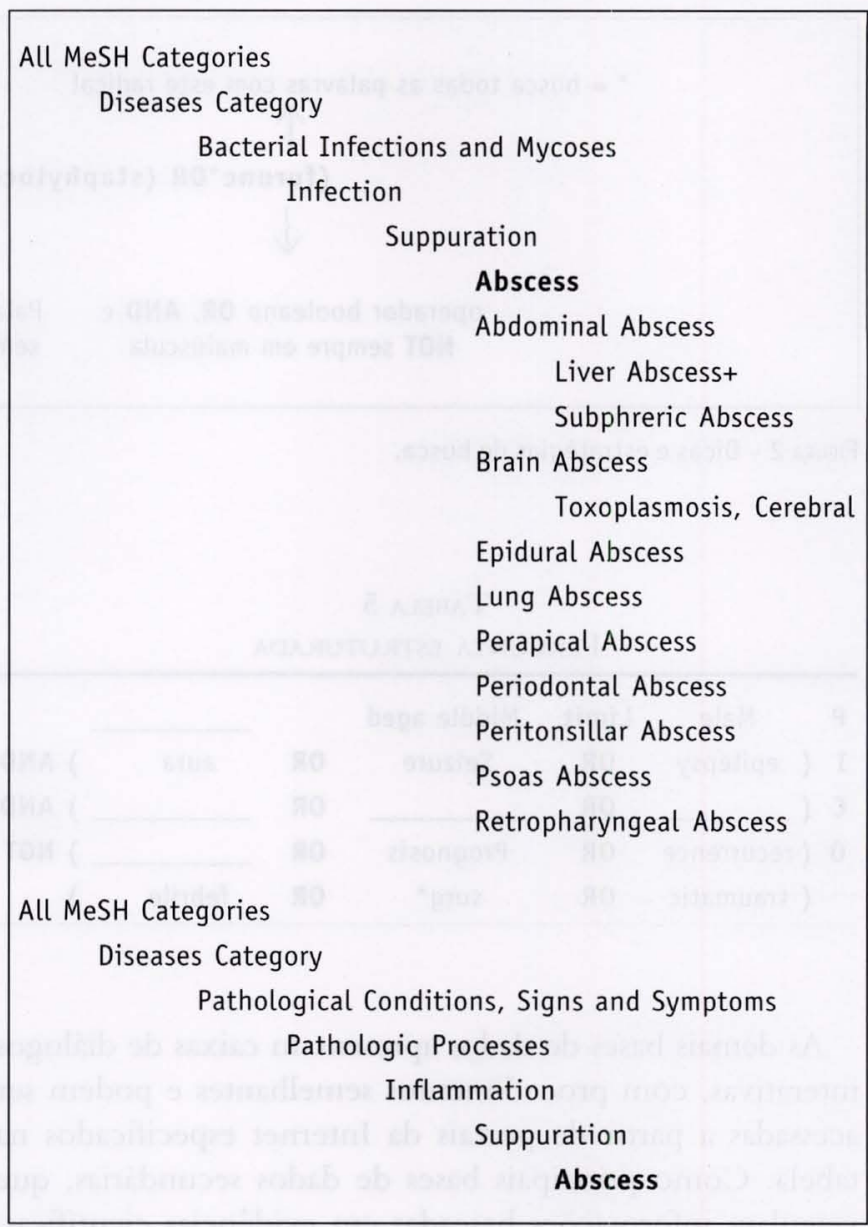

Figura 1 - Exemplo de árvore classificatória do MeSH - Pubmed.

TABELA 4

Estratégias de busca no Medline-Pubmed e na Cochrane
Encontra estudos que contêm um ou outro descritor, por exemplo: (child OR adolescent) seleciona tanto os com "crianças" como os com "adolescentes"
AND Encontra estudos que contêm os dois descritores, por exemplo, (child AND adolescent) seleciona somente os que têm os dois, "crianças" e "adolescentes"
NEAR Como o operador booleano AND, junta os descritores, mesmo que estejam separados por no máximo cinco palavras
NOT Exclui os estudos que contenham o descritor, por exemplo, (child NOT adolescent) seleciona somente os que têm "crianças"
Limits Coloca diferentes filtros que fazem diferentes restrições, por exemplo, data de publicação, língua inglesa, só palavras do título
( ) Agrupa palavras, por exemplo, (child OR adolescent) AND (hearing OR auditory) seleciona trabalhos com a palavra audição escrita de qualquer das duas maneiras tanto em crianças como em adolescentes
* Aceita qualquer palavra que contém o radical truncado, por exemplo child* = "child" ou "childs" ou "children" ou "childhood". Em outros bancos de dados o símbolo de truncagem é o \$ (cifrão)
[ti] ou :ti Seleciona estudos com os descritores no título, no Medline usa-se [ti] na Cochrane :ti
[so] ou :so Recupera artigos de determinada fonte, por exemplo, hearing AND BMJ [so] seleciona artigos sobre audição na revista BMJ
MeSH Acrônimo de Medical Subject Headings classifica os artigos segundo as palavras-chaves. Usado no Medline e Cochrane, torna a busca mais especifica. Exemplo, epilepsy busca artigos com a palavra no texto e título "Epilepsy" [MeSH] busca artigos classificados com esta palavra-chave, muitas vezes é útil usar as duas formas 


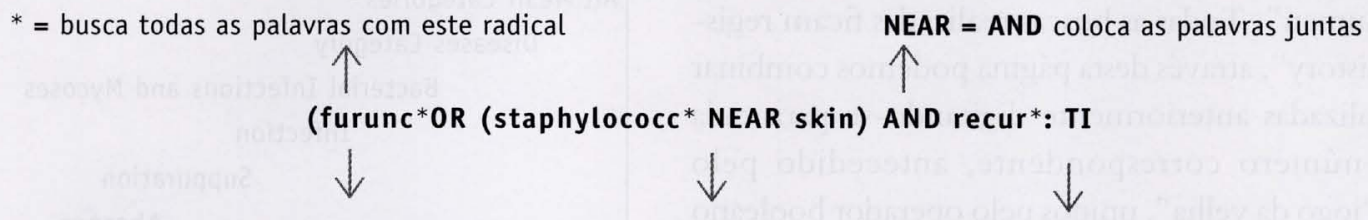

operador booleano OR, AND e

Palavras compostas NOT sempre em maiúscula os descritores devem estar no título

FiguRA 2 - Dicas e estratégias de busca.

TABELA 5

Pergunta estruturada

\begin{tabular}{|c|c|c|c|c|c|c|}
\hline $\mathbf{P}$ & Male & Limit & Middle aged & & & \\
\hline I & ( epilepsy & OR & Seizure & $\mathbf{O R}$ & aura & ) AND \\
\hline C & & OR & & $\mathbf{O R}$ & & -) AND \\
\hline 0 & (recurrence & $\mathbf{O R}$ & Prognosis & OR & & -) NOT \\
\hline & (traumatic & OR & surg* & OR & febrile & ) \\
\hline
\end{tabular}

As demais bases de dados apresentam caixas de diálogos interativas, com procedimentos semelhantes e podem ser acessadas a partir de portais da Internet especificados na tabela. Como principais bases de dados secundárias, que veiculam informações baseadas em evidências científicas, podemos citar: ACP Journal Club, Clinical Evidence e InfoPoems, respondendo questões de "foreground", Clinical Evidence e UpToDate, respondendo questões de "background" e "foreground", e ainda a Cochrane Library, disponibilizando revisões sistemáticas. Estas bases identificam estudos que contam com critérios de validade metodológica e de aplicabilidade clínica, os quais são veiculados em associação a comentários que posicionam o estudo em uma perspectiva clínica.
Outras informações sobre a prática clínica baseada em evidências podem ser obtidas nas Tabelas 6 e 7 ou através dos nossos endereços eletrônicos:

diretrizes@amb.org.br,mrcnobre@usp.br

wanderleybernardo@hotmail.com.

\section{TABELA 6}

PORTAIS DA INTERNET PARA ACESSO ÀS BASES DE DADOS PRIMÁRIAS E SECUNDÁRIAS E AOS PERIÓDICOS EM TEXTO INTEGRAL

\begin{tabular}{ll}
\hline Capes *1 & http://www.periodicos.capes.gov.br/ \\
Scielo *2 & http://www.scielo.br \\
SIBiNet-USP & http://www.usp.br/sibi \\
BVS-Bireme & http://www.bireme.br/bvs/P/pbd.htm \\
BioMed Central & http://www.biomedcentral.com/ \\
Free Medical Journals & http://www.freemedicaljournals.com/ \\
Ovid *3 & http://www.ovid.com/ \\
Science Direct & http://www.sciencedirect.com/
\end{tabular}

*1 acesso a partir de instituições de ensino superior, composto por teses e dissertações nacionais, e mais de 1.600 títulos de periódicos nacionais e internacionais em texto completo.

*2 publicação eletrônica cooperativa de mais de 100 periódicos científicos brasileiros, acesso público.

*3 apresenta filtro "EBM Reviews" que seleciona artigos revisados pela metodologia da Medicina Baseada em Evidência

TABELA 7

Coletâneas, livros e centros sobre Medicina Baseada em Evidências

$\begin{array}{ll}\text { Evidence-Based Medicine: How to Practice and Teach EBM } & \text { http://www.churchillmed.com } \\ \text { Clinical Evidence } & \text { http://www.evidence.org/index-welcome.htm } \\ \text { Bandolier } & \text { http://www.jr2.ox.ac.uk/bandolier/index.html } \\ \text { Centre for Evidence-Based Medicine - Oxford } & \mathrm{http://www.cebm.net/} \\ \text { Centre for Evidence-Based Medicine -Toronto } & \mathrm{http://www.cebm.utoronto.ca/} \\ \text { UpToDate } & \mathrm{http://www.uptodateinc.com/} \\ \text { Diagnostic Strategies for Common Medical Problems } & \text { http://www.acponline.org/catalog/books diagnostic_strategies_toc.htm }\end{array}$




\section{REFERÊNCIAS}

1. Nobre MR, Bernardo WM, Jatene FB. A prática clínica baseada em evidencias. Parte I - Questões clínicas bem construídas. Rev Assoc Med Bras 49(4):445-9, 2003.

2. Antman EM, Lau J, Kupelnick B, Mosteller F, Chalmers TC. A comparison of results of meta-analyses of randomized control trials and recommendations of clinical experts. Treatments for myocardial infarction. JAMA 268:240-8, 1992.

3. Hunt DL, Jaeschke R, McKibbon KA. Users' guides to the medical literature: XXI. Using electronic health information resources in evidence-based practice. Evidence-Based Medicine Working Group. JAMA 283:1875-9, 2000.

4. Sackett DL, Straus S, Richardson S, Rosenberg W, Haynes RB. Evidence-based medicine: how to practice and teach EBM. $2^{\text {a }}$ ed. Londres: Churchill Livingstone; 2000. p.4.

5. Musicco M, Beghi E, Solari A, Viani F. Treatment of first tonicclonic seizure does not improve the prognosis of epilepsy. First Seizure Trial Group(FIRST Group). Neurology 49(4):991-8, 1997. 